\title{
Análisis de la trayectoria académica del programa educativo de Ingeniería en Electrónica de la Facultad de Ciencias Químicas e Ingeniería
}

\section{Analysis of the academic path of the Electronic engineering educational program of the Chemical Sciences and Engineering School}

REYES-MARTÍNEZ, Roberto Alejandro†*, TRUJILLO-TOLEDO, Diego Armando, ESQUEDAELIZONDO, José Jaime y MIRANDA-PASCUAL, María Elena

Universidad Autónoma de Baja California, Facultad de Ciencias Químicas e Ingeniería

ID $1^{\mathrm{er}}$ Autor: Roberto Alejandro, Reyes-Martínez / ORC ID: 0000-0003-2210-2692, CVU CONACYT ID: 21470

ID $1^{\text {er }}$ Coautor: Diego Armando, Trujillo-Toledo / ORC ID: 0000-0003-1482-8581, Researcher ID Thomson: M-27702018M, CVU CONACYT ID: 232755

ID $2^{\text {do }}$ Coautor: José Jaime, Esqueda-Elizondo / ORC ID: 0000-0001-8710-8978, Researcher ID Thomson: I-2941-2017, CVU CONACYT ID: 90966

ID $3^{\text {er }}$ Coautor: María Elena, Miranda-Pascual / ORC ID: 0000-0002-9469-3383, CVU CONACYT ID: 455846

DOI: $10.35429 /$ JOTE.2020.13.4.24.31

Recibido 03 de Septiembre, 2020; Aceptado 05 de Diciembre, 2020

\section{Resumen}

Las Instituciones de Educación Superior implementan programas de estudio con el objetivo de formar profesionales competitivos que se ajusten a las necesidades del mercado laboral pero buscando en la medida de lo posible garantizar un egreso exitoso, es para ello necesario identificar las características de egreso de un programa educativo que permita a los gestores institucionales proponer estrategias que mejoren los niveles de egreso en los mismos y con ello mejorar las condiciones de calidad ofrecidas a los alumnos inscritos en dichos programas de estudio. Para poder llevar a cabo este proceso, se analiza desde el ingreso, el índice de reprobación, las condiciones del entorno escolar, los resultados de los exámenes de egreso de licenciatura y las acreditaciones del programa educativo. Esto se hace para identificar factores claves del proceso de deserción y en donde trabajar para la mejora del programa educativo de Ingeniería en Electrónica de la Facultad de Ciencias Químicas e Ingeniería de la Universidad Autónoma de Baja California.

Análisis, Trayectoria Académica, Ingeniería

\begin{abstract}
Higher Education Institutions implement study programs with the aim of training competitive professionals that adjust to the needs of the labor market, but seeking as far as possible to guarantee a successful graduation. It is necessary to identify the characteristics of graduation of a program education that allows institutional managers to propose strategies that improve graduation levels in them and thereby improve the quality conditions offered to students enrolled in these study programs. In order to carry out this process, the failure rate, the conditions of the school environment, the results of the bachelor's exit exams and the accreditations of the educational program are analyzed from the beginning. This is done in order to identify key factors in the dropout process and where to work for the improvement of the Electronic Engineering educational program of the Faculty of Chemical Sciences and Engineering of the Autonomous University of Baja California.
\end{abstract}

Analysis, Academic Trajectory, Engineering

Citación: REYES-MARTÍNEZ, Roberto Alejandro, TRUJILLO-TOLEDO, Diego Armando, ESQUEDA-ELIZONDO, José Jaime y MIRANDA-PASCUAL, María Elena. Análisis de la trayectoria académica del programa educativo de Ingeniería en Electrónica de la Facultad de Ciencias Químicas e Ingeniería. Revista de Educación Técnica. 2020, 4-13: 2431

*Correspondencia al Autor (correo electrónico: roberto@uabc.edu.mx)

$\dagger$ Investigador contribuyendo como primer autor. 


\section{Introducción}

En México, como en muchos países del mundo, la calidad de la educación es un tema ampliamente tratado a nivel pregrado (Castillo Ramírez et al., 2013; Elena et al., 2015; Martínez-Padilla \& Pérez-González, 2008; Núñez Cortés \& Errázuriz Cruz, 2020; PeláezValencia et al., 2019) y principalmente se refiere a la evaluación de pares externos que identifican que se cumple con una serie de criterios que permiten valorar la calidad del programa educativo evaluado (Loria, 2002).

Los Comités Interinstitucionales para la Evaluación de la Educación Superior (CIEES) surgieron en México en 1991 por una necesidad de aseguramiento de la calidad de los programas educativos y de las instituciones de educación superior (IES). Su misión es promover el mejoramiento de la educación superior mediante evaluaciones externas, pertinentes, válidas y confiables de los programas educativos y de las IES, así como reconocer su calidad (CIIES, 2019)

En el año 2000 se creó el Consejo para la Acreditación de la Educación Superior A.C. (COPAES) (COPAES, 2020) siendo el órgano regulador de las organizaciones acreditadoras, que para el área de la ingeniería se encuentra definido el Consejo de Acreditación de la Enseñanza en la Ingeniería (CACEI) (CACEI, 2020) que inició funciones desde 1994, pero no fue hasta el año 2000 que al integrarse al COPAES tuvo gran aceptación en las instituciones de educación superior para la revisión de la calidad de sus programas de ingeniería, entre sus funciones principales está el "contribuir al mejoramiento de la calidad del ejercicio profesional de la ingeniería" (Ocampo, 1994) mediante el establecimiento de sistemas de acreditación.

La Universidad Autónoma de Baja California (UABC) desde 1994 ha buscado que sus programas educativos sean evaluados de manera diagnóstica por CIEES o COPAES, para garantizar a su estudiantado que se encuentran en programas educativos de buena calidad (Moctezuma et al., 2013).
Un rubro de gran trascendencia en las evaluaciones es el análisis referente a los alumnos en el contexto de trayectoria académica (Bravo Guerrero et al., 2020), el cómo se forma en términos del tipo de educación (teórica, práctica, con proyectos o evaluaciones, así como otros factores), el cómo se desarrolla en el contexto particular a su carrera y de manera global, así como el acompañamiento que lleva a lo largo de su carrera, para garantizar un egreso en el programa de estudio y finalmente los resultados en evaluaciones globales a las que se somete.

Es ante un proceso de reacreditación de la calidad del programa educativo (PE) de Ingeniería en Electrónica (IE) de la Facultad de Ciencias Químicas e Ingeniería (FCQI) que se identifica la oportunidad de llevar a cabo un análisis de la trayectoria de su estudiantado, que permita conocer la evolución de factores que influyen en su ingreso, permanencia y egreso del PE (Bravo Guerrero et al., 2020).

Para ello en este artículo se ha desarrollado información estadística que se relaciona para identificar las áreas de oportunidad donde se debe trabajar para mejorar la trayectoria del estudiante dentro del PE de IE. En general, el artículo se presenta de la siguiente manera: en la sección de metodología se indica el proceso llevado a cabo, posteriormente en la sección de resultados mostrará las tablas y gráficas que dan representatividad al estudio de la trayectoria en los alumnos del PE de IE y permite identificar qué puntos son claves trabajar para mejorar la trayectoria de los alumnos; finalmente, se presentará una serie de conclusiones al respecto.

\section{Metodología}

Por el proceso de reacreditación llevado a cabo en este año por el PE de IE, se tiene la concentración de información clave para poder hacer un análisis estadístico que permita identificar si el rubro de trayectoria escolar ha mejorado o no y si existe una mejora, esta haya sido sustantiva.

Como contexto, el PE de IE de la FCQI ha participado en procesos de evaluación de la calidad educativa desde 1999, que se sometió a la revisión del comité de Ingeniería y Tecnología de los CIEES, quedando en nivel 1 en dicha evaluación.

REYES-MARTÍNEZ, Roberto Alejandro, TRUJILLO-TOLEDO, Diego Armando, ESQUEDA-ELIZONDO, José Jaime y MIRANDA-PASCUAL, María Elena. Análisis de la trayectoria académica del programa educativo de Ingeniería en Electrónica de la Facultad de Ciencias Químicas e Ingeniería. Revista de Educación Técnica. 2020 
Posteriormente en el 2005 se somete a la evaluación del COPAES a través del CACEI, obteniendo una evaluación favorable que fue refrendada en los años 2011, 2016 y actualmente se está en el trabajo para mantener un PE acreditado del 2021 - 2026. Además, en este mismo contexto se cuenta con un indicador de la calidad de sus egresados, al ser todos evaluados en su último semestre por medio del Examen General para el Egreso de Licenciatura (EGEL), referente nacional que maneja el Centro Nacional de Evaluación (CENEVAL) siendo un instrumento que mide los conocimientos y habilidades de los potenciales a egresar o recién egresados que le permiten iniciarse eficazmente en el ejercicio de su profesión (CENEVAL, 2020), este examen se ha presentado de manera periódica desde el 2005 a todos los potenciales a egresar del PE, del cual se ha presentado buenos resultados en el desempeño de los estudiantes, al tener en casi todas las aplicaciones más del $50 \%$ de estudiantes acreditados y desde el 2011 se ha mantenido dentro del padrón de alto desempeño (generalmente en Nivel 2).

Lo anterior pudiera dar una idea de que se cuenta con un programa de excelencia y que el análisis de trayectoria no debería ser un factor necesario a revisar o que se cuenten con áreas de oportunidad; sin embargo, con la información recopilada se ha identificado que si es necesario llevar a cabo un análisis más profundo.

Para este estudio, primero se revisó la eficiencia terminal, de acuerdo a la ecuación 1. donde se identificó el comportamiento entre el ingreso y el egreso dentro de los lineamientos institucionales.

$$
\% \text { EficienciaTerminal }=\frac{\text { Total } \text { Egreso }}{\text { Total } \text { Ingreso }} \times 100
$$

El ingreso a carrera se hace a partir del 3er semestre debido a que, durante el primer año los alumnos se encuentran en un programa denominado Tronco Común de las Ingenierías y al concluirlo, pueden decidir a qué programa de estudios ingresar de los 11 programas educativos del área de Ingeniería que se imparten en la universidad. Para el caso de la FCQI, sólo se ofertan los programas educativos de Ingeniería Química, Ingeniería en Computación, Ingeniería en Electrónica e Ingeniería Industrial.
$\mathrm{Si}$ el alumno quiere una carrera diferente, hace cambio a otra Unidad Académica de UABC que lo imparta sin ningún problema.

El promedio de ingreso por semestre, durante los últimos 6 años analizados y que garantizan contar con alumnos ya egresados, es de un grupo de 27 alumnos/semestre interesados en el programa educativo de Ingeniería en Electrónica, pero el indicador obtenido nos lleva a que sólo el $43 \%$ en promedio está concluyendo su carrera, identificándose que este bajo porcentaje lo genera principalmente el egreso de los últimos semestres evaluados, identificando que existen alumnos que todavía no concluyen los créditos obligatorias, ya que el alumno puede hacer uso de hasta 14 semestres en total para poder egresar de acuerdo a las condiciones estatutarias de los PE de la UABC (Estatuto Escolar de la Universidad Autónoma De Baja California, 2018.), con lo que se espera que en próximos semestres se vea mejorado el indicador, pero no en una gran medida.

Con base en la revisión de egreso se identifica otro indicador que se evaluó, siendo este el de la deserción para identificar las causas por las que el estudiante deja de estudiar en el PE de IE y busca otras opciones, así mismo buscando el indicador de reprobación para detectar cuales son las unidades de aprendizaje con mayores problemas y analizar si existe una correlación entre la deserción y la reprobación (Moreno \& Chiecher, 2019; Salinas et al., 2018).

Sobre el indicador de deserción, se presenta en la sección de resultados una tabla donde se identifica a los alumnos que no concluyeron sus estudios, y además se identifica el tipo de Baja que tuvieron, la cual puede ser: Baja Académica o Baja por Estatuto. La primera puede deberse a dejar un periodo o más sin avanzar en sus estudios, hacer cambio de carrera o cambio de universidad, el haber excedido el número de veces permitido que pueden reprobar una materia en el PE y, por lo tanto, ya no tiene derecho a seguir en el mismo o porque simplemente decidió su cambio al no sentirse a gusto en el PE. Por lo anterior, este tipo de baja abre la posibilidad de que no todos los que aparezcan en este estatus, ya no continúen en el PE, volviendo complicado un análisis más preciso sin alguna otra información que la numérica.

REYES-MARTÍNEZ, Roberto Alejandro, TRUJILLO-TOLEDO, Diego Armando, ESQUEDA-ELIZONDO, José Jaime y MIRANDA-PASCUAL, María Elena. Análisis de la trayectoria académica del programa educativo de Ingeniería en Electrónica de la Facultad de Ciencias Químicas e Ingeniería. Revista de Educación Técnica. 2020 
Por otro lado, la Baja por Estatuto es la que se presenta por haber alcanzado el límite de semestres y no haber concluido la carrera, dicha situación ha sido variable a lo largo de los semestres, pero se ha notado que en algunos semestres se alcanza casi el 50\% de este tipo de bajas, lo cual debe ser un punto de análisis de gran importancia para el PE.

El indicador de Reprobación se manejó en relación a los alumnos que abandonaron sus estudios buscando la existencia de una relación entre el desempeño académico y la consideración de no concluir en el PE. En la Tabla 4 de la siguiente sección se manejan tres situaciones: la primera en términos de la reprobación de una unidad de aprendizaje, la segunda con dos unidades de aprendizaje reprobadas y la tercera al haber reprobado tres unidades de aprendizaje o más.

Con esta información, se identificó el gran índice de reprobación que tienen los alumnos en el PE de IE, donde una gran cantidad de ellos tiende a reprobar tres unidades de aprendizaje durante su trayectoria, y posiblemente más. Esto repercute en que esos alumnos extiendan su egreso al repetir la unidad de aprendizaje reprobada poniendo en riesgo su conclusión con base al estatuto. Es por ello que también se revisó la tendencia del rezago escolar apoyado por los reportes del Sistema Institucional de Evaluación donde se presenta que un $38 \%$ del rezago se presenta en los primeros 3 semestres, incrementándose en la etapa disciplinaria a un $43 \%$ promedio, siendo el 4to semestre el que genera mayor rezago con el $50 \%$, mientras que en la etapa terminal se reduce significativamente al $27 \%$.

También, el gran índice de reprobación ocasiona que se corra el riesgo de que al volver a reprobar la unidad de aprendizaje tiendan a la Baja Académica. Para ello, se llevó a cabo un análisis de las materias con mayor índice de reprobación por periodo escolar a partir del 2015, donde a pesar de que no existe una unidad de aprendizaje recursiva todos los periodos, si hay unidades de aprendizaje con alta repetibilidad $y$ alto porcentaje de reprobación. Estas tienden a ser aquellas donde su contenido es ampliamente matemático o aquellas que requieren de un análisis con base a la identificación de una metodología a implementar para su desarrollo y principalmente de la etapa básica o disciplinaria de la carrera.

ISSN-2523-2460

ECORFAN $^{\circledR}$ Todos los derechos reservados
Con base a esta metodología planteada se obtuvieron los siguientes resultados.

\section{Resultados}

Siguiendo el orden llevado a cabo en la presentación de la metodología se presenta la Tabla 1 donde se indica los procesos de acreditación que se han tenido en el PE de IE

\begin{tabular}{|l|l|l|}
\hline Año & \multicolumn{1}{c|}{$\begin{array}{c}\text { Organismo } \\
\text { Acreditador }\end{array}$} & \multicolumn{1}{c|}{ Estatus } \\
\hline 1999 & CIEES & Nivel 1 \\
\hline 2006 & CACEI & Acreditado \\
\hline 2011 & CACEI & Acreditado \\
\hline 2016 & CACEI & Acreditado \\
\hline 2021 & CACEI & En proceso de Evaluación \\
\hline
\end{tabular}

Tabla 1 Procesos de Evaluación de la Calidad del PE de IE por organismos externos

Fuente: Elaboración Propia

Aquí se puede apreciar que el programa de Ingeniería en Electrónica lleva más de 20 años siendo evaluado como un programa de calidad, lo que indica que el rubro de trayectoria escolar se cumple dentro de los estándares evaluados.

Por otro lado, los alumnos que concluyen su carrera previamente realizan el EGEL, de donde se obtiene la Tabla 2 con base a los resultados obtenidos en dicho examen dentro del año evaluado y que son considerados dentro del Programa de Alto Rendimiento Académico (IDAP). Se identifica que los alumnos del PE han cumplido desde su participación en el mismo (2011) con resultados que los han mantenido dentro del Padrón de Alto Rendimiento $y$ ha sido para cada generación un compromiso mantenerse en este padrón y por tanto se muestra que el egreso del PE de IE en cuanto a conocimientos y habilidades que se requieren para su desempeño profesional, es un egreso altamente eficiente.

\begin{tabular}{|l|l|r|}
\hline \multicolumn{1}{|c}{ Convocatoria } & \multicolumn{1}{c|}{ EGEL } & $\begin{array}{c}\text { Nivel de } \\
\text { Rendimiento } \\
\text { Académico }\end{array}$ \\
\hline Julio 2011-junio 2012 & Ingeniería Electrónica & 1 \\
\hline Julio 2012-junio 2013 & Ingeniería Electrónica & 2 \\
\hline Julio 2013-junio 2014 & Ingeniería Electrónica & 1 \\
\hline Julio 2014-junio 2015 & Ingeniería Electrónica & 1 \\
\hline Julio 2015-junio 2016 & Ingeniería Electrónica & 2 \\
\hline Julio 2016-junio 2017 & Ingeniería Electrónica & 2 \\
\hline Julio 2017-junio 2019 & Ingeniería Electrónica & \\
\hline
\end{tabular}

Tabla 2 Resultados del IDAP del PE de IE Fuentepropia con base a la información plasmada en el reporte de resultados históricos del IDAP en CENEVAL) 
Los resultados anteriores podrían indicar que el PE de IE es un programa que se encuentra arriba del estándar y que el indicador de trayectoria escolar no es un problema, pero una vez recabada la Tabla 3 donde muestra la eficiencia terminal del programa es posible indicar que existen áreas de oportunidad y mejoras en la trayectoria escolar de los estudiantes.

De esta tabla se puede apreciar que, aunque el egreso es en promedio del $44 \%$, se considera sigue siendo un egreso bajo y que las bajas académicas son muy altas cercanas al $27 \%$ y pudiéndose incrementar en los años que todavía no concluye el egreso en un $13 \%$ más, lo que representa que este tipo de bajas tiene que analizarse desde otra perspectiva, para poder reducirse y mejorar el egreso. Así mismo, con esta tabla se aprecia que el 6\% de los alumnos se pierden porque alcanzan el máximo número de semestres en el $\mathrm{PE}$, este último dato debería bajarse a casi cero si se lleva un mejor programa de tutorías que dé seguimiento a la trayectoria escolar e identifique con tiempo la implementación de acciones que le permita a los estudiantes no caer en esta situación.

\begin{tabular}{|c|c|c|c|c|c|}
\hline 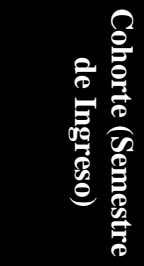 & 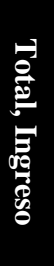 & 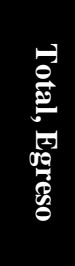 & 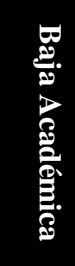 & 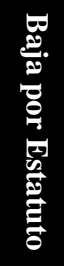 & 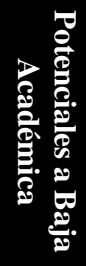 \\
\hline 2010-1 & 4 & 1 & 14 & 2 & \\
\hline $2010-2$ & 37 & 20 & 9 & 8 & \\
\hline 2011-1 & 24 & 8 & 3 & 3 & \\
\hline $2011-2$ & 32 & 15 & 11 & 3 & \\
\hline $2012-1$ & 13 & 7 & 11 & 6 & \\
\hline $2012-2$ & 41 & 26 & 16 & & 2 \\
\hline 2013-1 & 27 & 10 & 6 & & 7 \\
\hline $2013-2$ & 42 & 21 & 6 & & 8 \\
\hline 2014-1 & 20 & 6 & 3 & & 1 \\
\hline 2014-2 & 45 & 25 & 10 & & 6 \\
\hline $2015-1$ & 10 & 2 & 3 & & 5 \\
\hline $2015-2$ & 38 & 8 & 2 & & 6 \\
\hline $2016-1$ & 15 & 1 & & & 7 \\
\hline Promedio & 27 & $44 \%$ & $27 \%$ & $6 \%$ & $13 \%$ \\
\hline
\end{tabular}

Tabla 3 Eficiencia terminal del PE de IE Fuente: Elaboración Propia

De la Tabla 4 se pueden relacionar los factores de bajas con el de reprobación. Mientras que se tiene un estimado del $46 \%$ de deserción, se identifica que el porcentaje de alumnos que reprueban tres o más unidades de aprendizaje en algún semestre de su estancia es muy alto $(71 \%)$.
Se identifica como factor clave en la decisión de un estudiante para considerar que no es apto para ser un Ingeniero en Electrónica, que llegue a cursar una unidad de aprendizaje el máximo de tres veces que establece el estatuto o que rebase el tiempo estatutario y termine en baja.

Además, como un elemento positivo, se puede apreciar que la alta reprobación de unidades de aprendizaje en los últimos semestres ha ido disminuyendo significativamente como se puede ver en el gráfico 1, que nos presenta que cada vez más alumnos acreditan sus unidades de aprendizaje.

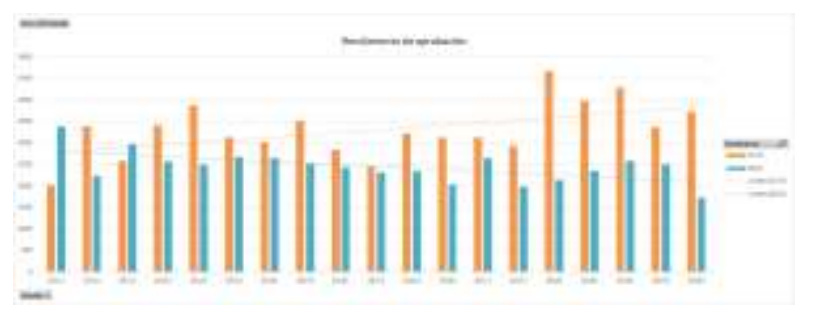

Gráfico 1 Rendimiento de aprobación en el PE de IE en el periodo 2015-2020

Fuente: Elaboración Propia

Las bajas académicas de un PE tienen diferentes vertientes, algunas se generan porque el alumno aún con lo visto en el tronco común, no tiene claro que involucra el estudiar la carrera de ingeniería en electrónica y cuando entra a la misma se da cuenta que no es lo esperado, por lo que tiende a reprobar, abandonar o cambiar de carrera, otro factor presente en la región es la necesidad de intercalar el estudio con trabajo, lo que reduce el tiempo dedicado a su carga académica e incrementa el riesgo de reprobación, pero también hay otro factor en donde se puede hacer algo desde el ámbito académico que es el de reprobación, el cual se presenta de una manera sencilla en la Tabla 4

\begin{tabular}{|c|c|c|c|c|c|c|}
\hline$\frac{\varrho}{\frac{\theta}{\theta}}$ & 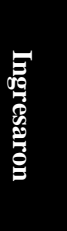 & 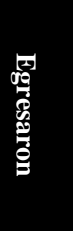 & 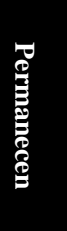 & 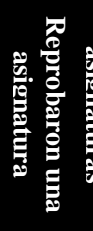 & 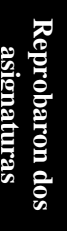 & 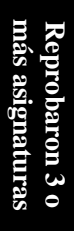 \\
\hline 20111 & 24 & 8 & & & 1 & 21 \\
\hline 20112 & 32 & 15 & & 2 & 1 & 21 \\
\hline 20121 & 13 & 7 & & 1 & & 11 \\
\hline 20122 & 41 & 26 & 1 & 1 & 1 & 35 \\
\hline 20131 & 27 & 10 & & 1 & & 25 \\
\hline 20132 & 42 & 21 & 3 & 3 & 1 & 36 \\
\hline 20141 & 20 & 6 & 1 & 2 & & 18 \\
\hline 20142 & 45 & 25 & 6 & 5 & 5 & 31 \\
\hline 20151 & 10 & 2 & 4 & & 1 & 9 \\
\hline
\end{tabular}

REYES-MARTÍNEZ, Roberto Alejandro, TRUJILLO-TOLEDO, Diego Armando, ESQUEDA-ELIZONDO, José Jaime y MIRANDA-PASCUAL, María Elena. Análisis de la trayectoria académica del programa educativo de Ingeniería en Electrónica de la Facultad de Ciencias Químicas e Ingeniería. Revista de Educación Técnica. 2020 


\begin{tabular}{|l|r|r|r|r|r|r|}
\hline 20152 & 38 & 8 & 14 & 4 & 3 & 27 \\
\hline 20161 & 15 & 1 & 6 & 1 & 1 & 13 \\
\hline 20162 & 30 & & 22 & 1 & 5 & 22 \\
\hline 20171 & 18 & & 11 & 1 & 1 & 16 \\
\hline 20172 & 22 & & 15 & 4 & 1 & 17 \\
\hline 20181 & 22 & & 17 & 1 & 2 & 19 \\
\hline 20182 & 33 & & 28 & 6 & 8 & 10 \\
\hline 20191 & 10 & & 10 & 4 & 2 & \\
\hline 20192 & 24 & & 24 & & & \\
\hline Totales & 466 & 129 & 162 & 37 & 33 & 331 \\
\hline
\end{tabular}

Tabla 4 Relación entre deserción y asignaturas reprobadas

Fuente: Elaboración Propia

Por otro lado, en la Tabla 5 se presenta la identificación de las 3 materias con mayor índice de reprobación por semestre, que muestra que, aunque no existe una recurrencia total de las materias a lo largo de todos los semestres, si hay algunas unidades de aprendizaje que destacan en la tabla por ser de manera regular y que se muestran en la Tabla 6.

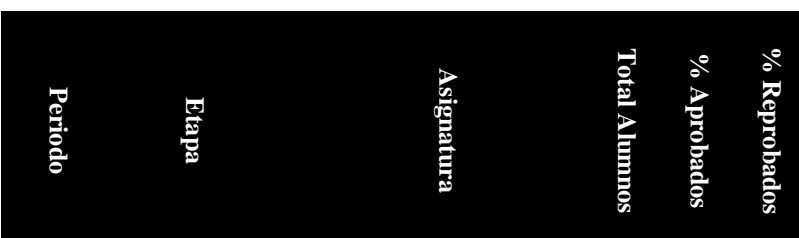

\begin{tabular}{|l|l|l|r|r|r|}
\hline $2015-1$ & Básica & Métodos Numéricos & 42 & 36 & 64 \\
\hline $2015-1$ & Básica & Circuitos Electrónicos & 44 & 36 & 64 \\
\hline $2015-1$ & Disciplinaria & Diseño Digital & 30 & 47 & 53 \\
\hline $2015-2$ & Disciplinaria & Electrónica de Potencia & 16 & 31 & 69 \\
\hline $2015-2$ & Disciplinaria & Comunicaciones & 11 & 36 & 64 \\
\hline $2015-2$ & Disciplinaria & Teoría Electromagnética & 15 & 47 & 53 \\
\hline $2016-1$ & Básica & Ecuaciones Diferenciales & 39 & 44 & 56 \\
\hline $2016-1$ & Básica & Circuitos Electrónicos & 44 & 52 & 48 \\
\hline $2016-1$ & Disciplinaria & Circuitos Eléctricos & 38 & 53 & 47 \\
\hline $2016-2$ & Disciplinaria & Comunicaciones & 15 & 33 & 67 \\
\hline $2016-2$ & Disciplinaria & Diseño Digital & 30 & 57 & 43 \\
\hline $2016-2$ & Disciplinaria & Señales y Sistemas & 28 & 61 & 39 \\
\hline $2017-1$ & Básica & Ecuaciones Diferenciales & 25 & 32 & 68 \\
\hline $2017-1$ & Disciplinaria & Acústica y Calor & 9 & 33 & 67 \\
\hline $2017-1$ & Disciplinaria & Modelado y Control & 39 & 51 & 49 \\
\hline $2017-2$ & Disciplinaria & Modelado y Control & 38 & 34 & 66 \\
\hline $2017-2$ & Básica & Circuitos Electrónicos & 33 & 48 & 52 \\
\hline $2017-2$ & Disciplinaria & Señales y Sistemas & 15 & 53 & 47 \\
\hline $2018-1$ & Disciplinaria & Circuitos Eléctricos & 25 & 8 & 92 \\
\hline $2018-1$ & Básica & Cálculo Multivariable & 30 & 37 & 63 \\
\hline $2018-1$ & Básica & Circuitos Electrónicos & 39 & 46 & 54 \\
\hline $2018-2$ & Básica & Circuitos Electrónicos & 32 & 41 & 59 \\
\hline $2018-2$ & Disciplinaria & Acústica y calor & 14 & 43 & 57 \\
\hline $2018-2$ & Disciplinaria & Circuitos Eléctricos & 39 & 56 & 44 \\
\hline $2019-1$ & Disciplinaria & Modelado y Control & 21 & 38 & 62 \\
\hline $2019-1$ & Básica & Ecuaciones Diferenciales & 25 & 40 & 60 \\
\hline $2019-1$ & Básica & Métodos Numéricos & 18 & 50 & 50 \\
\hline $2019-2$ & Disciplinaria & Microcontrola-dores & 25 & 44 & 56 \\
\hline $2019-2$ & Disciplinaria & Control avanzado & 11 & 45 & 55 \\
\hline $2019-2$ & Disciplinaria & Diseño analogico & 32 & 53 & 47 \\
\hline $2020-1$ & Disciplinaria & Modelado y Control & 32 & 28 & 72 \\
\hline $2020-1$ & Disciplinaria & Optoelectrónica & 9 & 33 & 67 \\
\hline $2020-1$ & Disciplinaria & Electrónica Analogica & 25 & 56 & 44 \\
\hline & & & & & \\
\hline
\end{tabular}

Tabla 5 Unidades de aprendizaje con mayor índice de reprobación periodo 2015 a 2020

Fuente: Elaboración Propia

\begin{tabular}{|c|c|c|c|}
\hline 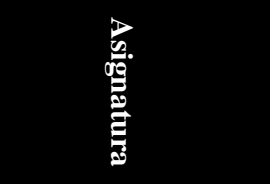 & 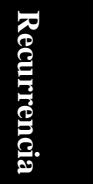 & 武 & 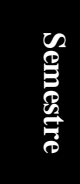 \\
\hline $\begin{array}{l}\text { Circuitos } \\
\text { Electrónicos }\end{array}$ & 5 & Básica & 3ero \\
\hline Modelado y Control & 4 & Disciplinaria & 5to \\
\hline $\begin{array}{l}\text { Ecuaciones } \\
\text { Diferenciales }\end{array}$ & 3 & Básica & 3ero \\
\hline Circuitos Eléctricos & 3 & Disciplinaria & 4to \\
\hline $\begin{array}{l}\text { Métodos } \\
\text { Numéricos }\end{array}$ & 2 & Básica & 3ero \\
\hline Diseño Digital & 2 & Disciplinaria & 4to \\
\hline Comunicaciones & 2 & Disciplinaria & 6 to \\
\hline Señales y Sistemas & 2 & Disciplinaria & 4to \\
\hline Acústica y Calor & 2 & Disciplinaria & 4 to \\
\hline
\end{tabular}

Tabla 6 Recurrencia de unidades de aprendizaje con mayor índice de reprobación periodo 2015 a 2020

Fuente: Elaboración Propia

Con base a la información de la Tabla 6 , se identifica que el 4to semestre es el semestre con mayor cantidad de materias que se encuentran entre las tres con mayor índice de reprobación, seguido del 3er semestre, lo que muestra una gran correlación con el índice de deserción en el primer año de su carrera (3er y 4to semestre) así como la posibilidad de que un alumno incremente su estadía por el PE cuando menos un año y con tendencia de hasta 3 años, llevando al alumno a alcanzar el límite de semestres para no concluir su egreso del PE.

Con todo lo anterior, se presenta en el gráfico 2 un análisis de fortalezas y debilidades de cómo se ha trabajado la trayectoria académica en el programa y una serie de temáticas que se deben abordar a nivel PE.

\begin{tabular}{|l|l|}
\hline \multicolumn{1}{|c|}{ Fortalezas } & \multicolumn{1}{c|}{ Oportunidades } \\
\hline - Programa Acreditado & - Incremento de la matrícula \\
\hline $\begin{array}{l}\text {-Alto Desempeño en el } \\
\text { EGEL }\end{array}$ & - Incremento del egreso \\
\hline - Programa de Tutorias & \multicolumn{1}{|c|}{-Apoyo de los profesores } \\
\hline \multicolumn{1}{|c|}{ Debilidades } & \multicolumn{1}{c|}{ Amenazas de la } \\
\hline -Alto índice de deserción & $\begin{array}{l}\text {-Disminución de } \\
\text { matrícula }\end{array}$ \\
\hline $\begin{array}{l}\text { - Falta de atracción por el } \\
\text { programa }\end{array}$ & -Apatía de los profesores \\
\hline $\begin{array}{l}\text {-Seguimiento reducido en } \\
\text { la tutoría }\end{array}$ & $\begin{array}{l}\text {-Mayor cantidad de } \\
\text { alumnos en bajas }\end{array}$ \\
\hline
\end{tabular}

Tabla 7 FODA sobre Trayectoria Académica en el PE de IE de la FCQI

Fuente: Elaboración Propia

En el PE de IE se cuenta con un programa acreditado, con egresados con gran desempeño en el examen EGEL. 
Sin embargo, existe una gran deserción mayor que la presentada comúnmente en el primer año de estudios de una carrera profesional y dentro de los diversos factores existentes, se ha considerado oportuno trabajar en los que puede competer a los miembros del PE de IE, siendo éstos presentados en la Tabla 7.

\begin{tabular}{|c|c|c|}
\hline $\begin{array}{l}\text { Problemá- } \\
\text { tica }\end{array}$ & Acción & Mejora \\
\hline $\begin{array}{ll}\text { Baja } & \text { por } \\
\text { estatuto } & \end{array}$ & $\begin{array}{l}\text { Mejorar en las tutorías el } \\
\text { seguimiento de los alumnos } \\
\text { con problemas de } \\
\text { reprobación, brindando un } \\
\text { acompañamiento } \\
\text { cercano }\end{array}$ & $\begin{array}{l}\text { Reducir a } 0 \text { en } \\
\text { un periodo de un } \\
\text { año las bajas por } \\
\text { estatuto }\end{array}$ \\
\hline $\begin{array}{l}\text { Alta } \\
\text { reprobación }\end{array}$ & $\begin{array}{l}\text { - Apoyar con asesorías las } \\
\text { unidades de aprendizaje de } \\
\text { 3er y 4to semestre } \\
\text { consideradas de alto índice } \\
\text { de reprobación. } \\
\text { - Presentar al alumnado una } \\
\text { nueva propuesta académica } \\
\text { semestral para cursar el 3er } \\
\text { y 4to semestre en tres } \\
\text { semestres }\end{array}$ & $\begin{array}{l}\text { Reducir al } 30 \% \\
\text { la reprobación }\end{array}$ \\
\hline $\begin{array}{l}\text { Bajo ingreso } \\
\text { a carrera }\end{array}$ & $\begin{array}{l}\text {-Promocionar el PE en el } \\
\text { alumnado de tronco común }\end{array}$ & $\begin{array}{l}\text { Incrementar el } \\
\text { ingreso } \\
\text { promedio en un } \\
10 \% \text { cada } \\
\text { semestre }\end{array}$ \\
\hline
\end{tabular}

Tabla 8 Propuesta de acciones para resolver los problemas de trayectoria escolar en el PE de IE

Fuente: Elaboración Propia

\section{Conclusiones}

En este artículo se presentó un análisis estadístico de elementos que repercuten de manera sistemática en la trayectoria académica de los alumnos del PE de IE y que a pesar de ser un programa exitoso en término de cumplir con estándares externos (acreditación) y estar a nivel nacional en el padrón de excelencia en relación al examen EGEL, se sigue teniendo los grandes problemas de retención a nivel licenciatura existentes en México. Al identificar con mayor claridad los niveles de deserción, unidades de aprendizaje reprobadas y materias con mayor índice de reprobación, se pudo ver una cierta correlación de estos elementos que influyen en la decisión de deserción del PE y que a través de un plan de acción simple que se instrumente adecuadamente, poder bajar en un $10 \%$ el índice de reprobación y que si se suman otros factores propios del estudiante que se pueden cambiar con las acciones que se plantean, posiblemente otra reducción del 5\%, lo que nos llevaría a condiciones que favorezcan más el egreso, sin sacrificar la calidad del programa mismo.

ISSN-2523-2460

ECORFAN $^{\circledR}$ Todos los derechos reservados

\section{Referencias}

Bravo Guerrero, F. E., Illescas-Peña, L., \& Peña Ortega, M. P. (2020). Trayectoria Académica de Estudiantes Universitarios. Podium, 37(37), 27-42. https://doi.org/10.31095/podium.2020.37.3

\section{CACEI. (2020). CACEI. http://cacei.org.mx/}

Castillo Ramírez, M., Landeta, I., Manuel, J., González, E., \& Erika, D. (2013). CPU-e, Revista de Investigación Educativa. CPU-e, Revista de Investigación Educativa, 17, 172188.

http://www.redalyc.org/articulo.oa?id=2831283 29007

CIEES. (2019). CIEES - Comités Interinstitucionales para la Evaluación de la Educación Superior. https://www.ciees.edu.mx/

COPAES. (2020).

COPAES.

https://www.copaes.org/

Elena, N., Betancur, H., \& Torres-Madronero, M. C. (2015). ACCREDITATION OF ENGINEERING PROGRAMS IN THE REGION: A COMPARATIVE ANALYSIS. Revista Educación En Ingeniería, 10(19), 8089. http://www.educacioneningenieria.org

ESTATUTO ESCOLAR DE LA UNIVERSIDAD AUTÓNOMA DE BAJA CALIFORNIA. (2018).

CENEVAL. (2020). Exámenes Generales para el Egreso de Licenciatura (EGEL) - Ceneval. https://www.ceneval.edu.mx/examenesgenerales-de-egreso

Loria, E. (2002). La competitividad de las universidades públicas mexicanas. Una propuesta de evaluación. Distrito Federal, México: Universidad Autónoma Del Estado de México y Plaza y Valdés.

Martínez-Padilla, J. H., \& Pérez-González, J. A. (2008). Efecto de la Trayectoria Académica en el Desempeño de Estudiantes de Ingeniería en Evaluaciones Nacionales. Formación Universitaria, $\quad 1(1), \quad 3-12$. https://doi.org/10.4067/s071850062008000100002 
Moctezuma, P., Ocegueda, M. T., Mungaray, A., Ocegueda, J. M., \& Estrella, G. (2013). Cobertura con calidad a través de la reorganización institucional en la educación superior de Baja California. Frontera Norte, 25(49), 87-108. http://www.scielo.org.mx/scielo.php?script=sci _arttext\&pid=S0187-

$73722013000100004 \& \operatorname{lng}=\mathrm{es} \& \mathrm{nrm}=\mathrm{iso} \& \operatorname{tn} \mathrm{l}=$ es

Moreno, J. E., \& Chiecher, A. C. (2019). Abandono en carreras de Ingeniería. Un estudio de los aspectos académicos, sociodemográficos, laborales y vitales. Cuadernos de Investigación Educativa, 10(2), 73-90. https://doi.org/10.18861/cied.2019.10.2.2908

Núñez Cortés, J. A., \& Errázuriz Cruz, M. C. (2020). Panoramas de la alfabetización académica en el ámbito iberoamericano: Aportes para la calidad de la Educación Superior. Tendencias Pedagógicas, 36, 1-8. https://doi.org/10.15366/tp2020.36.01

Ocampo, J. F. (1994). La acreditación y su relación con la calidad de la enseñanza. Revista Ingeniería Civil, 307.

Peláez-Valencia, L. E., Trefftz, H., Iván, \&, \& Delgado-González, A. (2019). Acreditación internacional de carreras de ingeniería • International accreditation program for engineering. Revista Educación En Ingeniería, 15(29), 28-33. https://doi.org/10.26507/rei.v15n29.1044

Salinas, W. E. A., de Las, M., Lara, F., Gonzalo, C., Monroy, I., \& Rivera Castellón, R. E. (2018). Perfiles de estudiantes asociados a las características de reprobación de las asignaturas de ciencias básicas en ingeniería. In Boletín Redipe, ISSN-e 2256-1536, Vol. 7, $N^{o}$. 8, 2018 (Ejemplar dedicado a: Ethics, management evaluation educational), págs. 129-145 (Vol. 7, Issue 8). REDIPE, Red Iberoamericana de Pedagogía. https://dialnet.unirioja.es/servlet/articulo?codig $\mathrm{o}=6523207$ \&info $=$ resumen\&idioma $=$ SPA 